\title{
Strategies for Tomography
}

\section{Preface}

\section{Introduction}

Inverse problems concerning image reconstruction of unaccessible objects are widely considered in various areas of research. For instance, in Computerized Tomography the target is represented by the interior of an X-ray scanned human body, and the reconstruction can be obtained through analytic or algebraic methods. In other applications of Tomography the goal is represented either by the knowledge of the chemical structure of a material or the investigation of its physical properties, such as refraction, diffraction, transmission. Construction of models for reducing the number of required projections is a research area where theory and applications interact. The areas of Geometric and Discrete Tomography play a special role here.

The Meeting on Tomography and Applications is an annual meeting organized since 2007, with the main purpose of providing a forum for mathematicians and experimentalists to discuss the state of the art of tomographic research. The Meeting is organized by the Department of Mathematics of Politecnico di Milano. The seventh edition, which took place on May 20-21,2013, was concerned with an overview of strategies employed in some of the X-ray tomographic investigations.

The papers appearing in this Special Issue went through a thorough refereeing process. Some of them discuss, in more details and depth, topics presented by the speakers in the corresponding talks, while others papers present original contributions.

The remainder of this preface consists of two parts. The first part gives a brief overview of each paper accepted for this Special Issue. The second part provides the summaries of the talks presented at the 7th Meeting on Tomography and Applications, with some references given for each contribution. We hope that, in this way, the reader can get a meaningful insight into the nature of the meeting as well as an overview of the current research in the areas covered by this volume. 


\section{Overview of the papers from this issue}

- L. Plantagie, K.J. Batenburg, Approximating algebraic tomography methods by filtered backprojection: a local filter approach.

The process of reconstruction of unknown discrete objects from a set of projections obtained by letting high energy beams cross the set with given incidence angles and successively measuring the obtained attenuations, relies mainly on two different approaches: the analytical, which is concerned with the (analytical) methods to compute the inverse of the discrete Radon transform (whose best known example is the Filtered Backprojection), and the algebraic, that considers the whole reconstruction process as the solution of an extremely undetermined system of linear equations, such as ART, SART, and SIRT. Both approaches have their advantages and drawbacks, resulting from the usual struggle between the speed of the process and the quality of the reconstructed image.

In this paper the authors explore the possibilities of merging these approaches: the information encoded in the system of equations is modelled using a series of local algebraic filters that cover the entire reconstruction grid, and then are used in the Filtered Backprojection. A similar approach, the AF-FBP, has been already studied by the authors, using one single algebraic filter.

An accurate experiment has been carried on, where the performances of one filter and multiple filters AF-FBP are compared in approximating the SIRT method. Interesting results were obtained in presence of Poisson noise in the projection data.

- F. Bleichrodt, J. de Beenhouwer, J. Sijbers, K.J. Batenburg, Approximate discrete reconstruction algorithm.

The reconstruction algorithms defined for Discrete Tomography often rely on models that consider exact projections data, i.e., data that are supposed to be acquired by ideal scanning devices, with no errors of detection, and acting exactly along a set of given angles. Unfortunately, such models have limited applications for high resolution microtomography or nanoscale imaging, where the stability of the scanner and the motions of the object limit the accuracy of the detections, and may lead to unaligned projection images. Most of the algorithms for aligning the projection data track specific points (markers) in the picture, but a markerless approach can also be used, mainly in applications at nano scales, where the cross-correlation between consecutive images with low angular separation is exploited.

In this paper, the authors propose a new method for the alignment of binary tomography data, called Discrete Tomography Projection Matching (DTPM), that splits the problem into a discrete tomography reconstruction algorithm and a minimization process for the determination of the alignment parameters. More precisely, the authors insert parameters inside the sensor matrix to take care of the motion instability of the real system (translation, rotations), and alternate estimation of parameters with a volume reconstruction approach based on DC programming. To minimize the matrix parameters they use a modified version of the Levenberg-Marquardt algorithm. A final experimental section demonstrates the accuracy advantages of using the tomography algorithm DC combined with a projection matching method for the relevant cases with low number of projections. In case of a large number of projections, the alignment method gives its best accuracy performances when combined with an algebraic reconstruction approach as in SIRT. 
- S. Brocchi, A New Approach for the reconstruction of object-Based images in Discrete Tomography.

The problem of the reconstruction of an unknown homogeneous object, regarded as a discrete set of points, from a series of projections along a fixed set of discrete directions, is considered to be an ill-posed problem, meaning that, in general, there may exist a huge class of discrete sets, which pairwise may also have no common points, compatible with the given projections. To overcome this problem, in research on a faithful reconstruction of the object different approaches have been proposed, with most of them using some a priori knowledge that has to be somehow inserted in the reconstruction process to guide it towards a smaller set of feasible solutions. In this paper the author follows such an approach and requires the unknown objects to be composed by a set of disks stuffed inside a solid ring. Such configurations have been already considered in the literature because of their high expressivity in modelling sections of biological tissues (like porosities inside a bone or breast metastasis), and more recently, in order to develop effective evolutionary reconstruction algorithms.

So, the author proposes an efficient reconstruction algorithm for this class of objects that first uses an analytical approach to detect the positions of the internal disks with a small margin of error, then it extracts relevant information about the object by examining very few directions of projections, and finally it reaches a full reconstruction of the object. The author makes benchmarks with previous similar algorithms providing an evidence of the remarkable precision and speed of the reconstruction process while identifying as a drawback its high sensibility to noise.

- S. Brlek, N. Lafreniere, On reconstructing words from its f-palindromic language.

The wide spectrum of problems that con be treated with a tomographical approach also includes some concerning molecular biology, and, in particular, DNA analysis. Indeed DNA is a doublestranded helix, composed by two long sequences of molecules called nucleotides (guanine, adenine, thymine, and cytosine), coded by their initial letter G, A, T, and C. So, each DNA sequence can be regarded as a word on the alphabet $\Sigma=\{A, C, G, T\}$, while the pairing relation between the single strand sequences can be expressed by the simple involution $\sigma: A \leftrightarrow T, C \leftrightarrow G$. The detection, and the reconstruction from partial and sometimes inaccurate information of specific patterns inside these paired words allows one to predict geometric properties of the secondary structure of the DNA. In particular, this paper concerns the definition of the Watson-Crick palindromes in terms of involutions, and their complexity factor, i.e., the information content that each palindrome of the class can encode.

First, the study of the complexity of palindromes and $\sigma$-palindromes words is carried on, and examples of saturated words and comparisons with other relevant classes of words are provided. The huge dimension of strands of nucleotides motivates the authors to extend their results to infinite classes of words defined from $\sigma$-palindromic languages. Finally, the obtained results allow to compute the $\sigma$-palindromic language of a generating word $w$, together with its $\sigma$-lacunas. The defined algorithm applies to finite $w$ only, but the authors suggest a possible generalization to infinite words having finite defect or finite $\sigma$-palindromic language. 
- A. Denitiu, S. Petra, C. Schnorr, Phase transitions and cosparse tomographic recovery of compound solid bodies from few projections.

The ill-posedness of the problem of reconstructing an unknown object from projections is usually limited by acquiring a priori information about the object, and then using it during the reconstruction process. This information ranges from global assumptions about the geometry of the object or the maximum number of its primary constituents to the very local knowledge of some of its elements. Here, the authors consider this problem from the broader viewpoint of compressive sensing and they explore the possibility of using its tools in the tomographical framework. Compressive sensing has been studied in depth for detecting general signals from very few samplings, under the assumptions that a signal is a sparse object that carries much less information than commonly used for its representation. So an adequate representation, and an efficient sampling of a signal could crucially condense its information in very few data. This viewpoint is applied to image reconstruction with the analogous assumption that images must be somehow compressible, so that few ad hoc samples could capture their degrees of freedom and enable the reconstruction.

More precisely, the paper deals with the reconstruction of $2 D$ or $3 D$ solid bodies from a small set of projections obtained by tomographic measurements, under the assumption that gradients of their related density functions are sufficiently sparse. The authors started with a theoretical approach to the possibility of unique recovery of a signal (image), using the concept of cosparsity, from undersampled linear systems. In the second part of the paper, they rely on the obtained results to provide a linear programming strategy to solve the unique recovery problem. Finally they support their study with a series of numerical experiments showing that the imposed cosparsity constraint performs well with random generated images.

- P. Balázs, N. Hantos, Random generation of hv-convex polyominoes with given horizontal projection.

It is a common consensus that Discrete Tomography differs from Computerized Tomography not in the problems they consider, but rather in the tools used for solving those problems. While the latter uses concepts mainly borrowed from mathematical analysis, the first relies on discrete geometry and combinatorics. Thus it is not surprising that geometrical studies of specific classes of discrete objects from a geometrical point of view leads both to their enumeration and to the definition of reconstruction from projections strategies. In this paper, the authors analyze the geometrical and combinatorial properties of a well known (and widely studied) class of discrete $2 D$ objects, viz., the polyominoes, that are defined as finite sets of connected points satisfying the horizontal and vertical convexity ( $h v$-convex polyominoes). After demonstrating that these objects are geometrically characterized by the superposition of two stack and a parallelogram polyominoes, a technique is presented for enumerating these two classes according to the number of cells on each line. It is remarkable that the two defined enumerations are based on a coding of each polyomino by an integer vector, so that the random generation of these vectors naturally leads to the random generation of a horizontal and vertical convex polyomino having a prescribed number of elements on each row in quadratic time w.r.t. the input vector of projections. We point out that, although several algorithms for the enumeration and reconstruction of $h v$-convex polyominoes from two projections (and for their uniform generation) were studied in the literature, quite surprisingly until now these studies have not been extended to one single direction of projection. 
- A. Goupy, S.M.C. Pagani, Probabilistic reconstruction of hv-convex polyominoes from noisy projection data.

This paper considers the class of $h v$-convex polyominoes, and in particular the problem of their reconstruction from the horizontal and vertical projections, whose entries could be affected by a random noise with its intensity bounded by a constant value. Such an approach leads to a probabilistic evaluation of the possibility of each single cell to be present in the reconstructed solution: the values of the evaluation may range from 0 (when no solution exists containing the cell) to 1 (when all the solutions of the noisy instance contain the cell) with the intermediate values giving rise to a grey level reconstruction. This setting is widely studied in Binary Tomography since, in practice, the detector devices often provide projections affected by appreciable error, and the low number of acquired projections usually do not contain enough information for a faithful reconstruction. Furthermore, theoretical results about the stability of the reconstruction process affirm that small errors in the projections may lead to completely different solutions.

Based on a series of previous studies that consider reconstruction strategies in case of exact projections, the authors extend them by including noise in the projections. They define an algorithm that iterates on the unidentified point of the minimal bounding rectangle including the polyomino, and computes the probabilistic value of at least one point, till no further detections are possible. Then, a priori information about the object is considered (viz., the exact location of one single point at a time) and the computation starts again. On the other hand, if no additional information is present, then the unidentified points can be arranged into sequences of switching components allowing multiple solutions.

\section{- R. A. Fiorini, Discrete Tomography Background Noise Minimization by CICT.}

In dealing with the inverse problem of tomographic image reconstruction, one often has to manage a very huge amount of collected data affected by some kind of noise. Consequently, the identification of the noise sources becomes crucial.

A new way of approaching this problem is based on a combinatorial approach, which leads to a characterization of real instrumentation structures which, reliably, can automatically profile artifact and noise as a whole.

The paper briefly surveys the noise problem in different areas where tomographic reconstruction methods are employed. This is then related to the classical approaches to noise in Information Theory, mainly based on the definition of Shannon entropy. Consequently, it is pointed out that it is usually almost impossible to discriminate the so called random noise from any possible combinatorially optimized coding message which is referred to as deterministic noise. An interesting example is presented, where the Shannon entropy for a grey image is computed in the case of an optimal combinatorial white noise source, showing that such an image is in fact indistinguishable from a corresponding instance generated by a random source noise. In order to solve this conundrum, it is proposed to introduce a new concept of entropy, called coherent Shannon entropy, by means of the Computational Information Conservation Theory (CICT). The first attempt is carried out for developing basic principles for machine noise sources characterization, profiling an identification from discrete system parameters by CICT. In particular, a Deterministic Noise Source Coherent Profiling procedure is presented, which should allow the development of explicit algo- 
rithms for either managing conservation of information or becoming aware of the dissipation of information in a competitive way.

- R. Fedele, A. Ciani, F. Fiori, X-ray microtomography under loading and 3D-Volume Digital Image Correlation. A Review.

The paper offers a review of the main advances in Computerized Tomography, starting from early $60 s$ to nowadays. It outlines the progress made in the reconstruction techniques from projections with respect to the accuracy of the reconstruction, the average time required by the process, the range of different situations and different materials of appliance, and the number of projections needed to gain a faithful output.

Since its beginnings, Computerized Tomography was mainly concerned with medical diagnostics and the health care, but then, due to its improvements with respect to the accuracy of reconstructions, it acquired relevance also in the study of material specimens and in quality control, after changing the setting's parameters.

Nowadays, the high accuracy of the tomographical devices reached by the third generation synchrotron radiation sources allows to reach micrometer resolutions, and both to investigate and to reconstruct millimeter-sized objects. Thus in this way the range of applications of the tomographical techniques move from biological specimens to all kinds of natural materials and industrial components.

The paper begins with presenting the basic characteristics of X-ray micro-tomography and the theoretical results for 3D-Volume Digital Image Correlation. Then it provides an overview of the potential that these two techniques are expressing when combined in a widely adopted tool for material characterization. After presenting different strategies for noise detection and control, a large section presents a wide spectrum of inspections inside biological, natural and industrial materials that have been carried on.

- M. Compagnoni, R. Notari, TDOA - based localization in two dimensions: the bifurcation curve.

One of the main problems in the wide area of signal processing concerns the localization of an object in space. The scenario presented in this paper consists of three detectors in the plane measuring the time difference of arrival (TDOA) of synchronized noiseless radio signals originating in an unknown point source. In literature, different methods have been developed to detect the source point, all of them relying on the fact that the geometrical properties of the signal propagation force the emitting point to lie on a curve parameterized with respect to each associate detector. Theoretical studies have shown that the non-linearity of these curves allows, in general, multiple feasible solutions that locate in the so called bifurcation set, which is a region that separates the two-solutions region and the one-solution region. In their previous studies, the authors provided a series of geometric properties of the region that lead to its partial characterization as an algebraic curve, without being able to determine the Cartesian polynomial equation.

In this paper, they complete the previous analysis by computing the explicit Cartesian equation of the bifurcation curve $E$ in terms of the positions of the detectors. The knowledge of this equation is a key point for the localization of the emitting source in its neighborhood, both in terms of the accuracy and the speed. The curve $E$ is obtained by first computing the polytope that is the 
intersection region of the TDOAs for each pair of detectors, and then determining the unique internal ellipse tangents to its sides. Finally, by Taylor expansion, one obtaines the explicit form of the real part of $E$ as a degree five equation.

\section{Outline of the talks presented during the Meeting on Tomography and Applications, Milano, May 20-21, 2013}

\section{Challenges in 4D tomography Joost Batenburg, Vision Lab, Dep. of Physics, University of Antwerp (CD)}

Time-resolved (4D) tomography aims to reconstruct a sequence of 3D images of an object, as it evolves over time.

In addition to three dimensional volumetric anatomical information, as in conventional CT, 4D-CT is able of resolving organ motions due to, for example, patient respiration. This is done by reconstructing a set of CT images corresponding to different respiratory phases in a breathing cycle.

The goal of improving temporal resolution directly conflicts with the goal of improving the reconstruction quality at a particular time, as fewer projections (or noisier projections) are available at that time point.

The key to accurate 4D image reconstruction is to exploit prior knowledge in both the spatial and temporal domains. Effective algorithms for 3D image reconstruction from limited data are now available (e.g. in discrete tomography and compressive sensing), but they do not capture the correlations between the measured data at different time points. The inclusion of a temporal dimension leads to new reconstruction problems and requires new algorithms.

In his talk Joost Batenburg introduced the fascinating topic of 4D tomographic reconstruction, through an overview of recent experimental developments. He then covered the mathematical problems that arise in $4 \mathrm{D}$ tomography. After discussing several solution strategies, the talk concluded with a demonstration of some recent results.

\section{References}

[1] Van Eyndhoven, G., Batenburg, K.J., and Sijbers, J., Region-Based Iterative Reconstruction of Structurally Changing Objects CT, IEEE Trans. Image Proc. 23(2), 2014, 909-919.

[2] Kazantsev, D., Lionheart, W.R.B., Withers, P.J., Lee, P.D., GPU accelerated 4D-CT reconstruction using higher order PDE regularization in spatial and temporal domains In: Uni of Salamanca, 13th International Conference Computational and Mathematical Methods in Science and Engineering. Spain, Almeria 2013/07/24. CMMSE 2013: Proceedings of the 13th International Conference on Mathematical Methods in Science and Engineering: ISBN: 978-84-616-2723-3.

[3] Hahn, B., Efficient algorithms for linear dynamic inverse problems with known motion, Inverse Prob. 30, 2014, 035008. 


\section{The identity transform of a permutation Daniela Battaglino, Universitá di Siena}

Some recent problems of Discrete Tomography rely on a quite popular theorem by Marshall Hall in 1952 [3], which states:

Theorem (Halls Theorem). Let $G=\left\{a_{1}, \ldots, a_{n}\right\}$ be an abelian additive group of order $n$ and let $c_{1}, \ldots, c_{n}$ be elements of $G$, not necessarily distinct. Then there is a permutation $p$ of $\left\{a_{1}, \ldots, a_{n}\right\}$ such that $a_{i}+p\left(a_{i}\right)=c_{i}$ for $i=1, \ldots, n$, where $c_{i}$ are reordered properly, if and only if

$$
\sum_{i=0}^{n} c_{i}=0
$$

The proof of Halls Theorem essentially consists of an algorithm which finds the desired permutation $p$. The algorithm runs in $O\left(n^{2}\right)$ time. Note that, in general, a solution to Halls Theorem, if it exists, is not necessarily unique.

No proof exists which is substantially different from the one proposed in [3]. Anyway, Halls Theorem is a fundamental result which lies at the bases of a popular research subject known under the name of zero-sum problems, fitting in the generic area known as Ramsey Theory.

Halls Theorem has been rediscovered many times (for instance by F.Salzborn and G.Szekeres [5]). However, all these proofs reduce to the one given by Hall himself, which is implicitly a non constructive proof, since it uses the reductio ad absurdum.

Alberto Del Lungo [1] discovered a generalization of Halls Theorem to multisets of cardinality $k_{n}$ having zero-sum. In our work we start a very general study with the main goal of giving a completely different proof of Halls Theorem.

After commenting briefly on Hall's Theorem, Daniela Battaglino defined the identity transform of a permutation $p$ as $C(p)=(0+p(0), 1+p(1), \ldots,(n-1)+p(n-1))$, and considered the set $C_{n}=\{(C(p)$ : $\left.p \in S_{n}\right\}$, where $S_{n}$ is the set of permutations of the elements of the cyclic group $Z_{n}$. Some closure properties of $C_{n}$ have been given together with some of its combinatorial and algebraic characterizations as well as connections with other combinatorial structures.

This leads to an algorithm for the exhaustive generation of the whole class $C_{n}$ and for the exhaustive generation of all the solutions of Hall's problem starting from a given vector having zero sum.

Finally, some links with other topics in discrete mathematics (e.g., with Latin squares) have been given.

\section{References}

[1] Del Lungo, A.: Reconstructing permutation matrices from diagonal sums, Theor. Comput. Sci. 281, 2002, 235-249.

[2] Erdös, P., Ginzburg, A.: Ziv, A., A theorem in additive number theory, Bull. Res. Council, Israel F 10, 1961, 41-43.

[3] Hall, M. Jr.: A combinatorial problem on abelian groups, Proc. Amer. Math. Soc. 3, 1952.

[4] Hall, M. Jr.: A survey of difference sets, Proc. Amer. Math. Soc. 7, No. 6, (1956).

[5] Salzborn, F., Szekeres G.: A problem in combinatorial group theory, Ars Combinatoria 7, 1979, 3-5. 


\section{A Geometric Model for Radiant Source Localization based on TDOA Marco Compagnoni, Politecnico di Milano}

The localization of a radiant point source is a common problem for many scientific research areas, such as seismology and astronomy, and it forms the basis for the development of technologies like GPS, radar and sonar devices. The talk presented a geometric model for localization. It is based on the measurements of time differences of arrival (TDOA) of the emitted signals between couples of distinct sensors. In particular, using multilinear algebra and (real) algebraic geometry techniques, it was shown how to completely solve the localization problem in the case of three synchronized and calibrated microphones lying in a plane. Marco Compagnoni also reported on the conditions for the existence and uniqueness of localization, and how to characterize the critical region for the reconstruction problem.

\section{References}

[1] Bancroft, S.: An Algebraic Solution of the GPS Equations, IEEE Trans. Aerosp. Electron. Syst. 21, 1985, 56-59.

[2] Bestagini, P., Compagnoni, M. , Antonacci, F., Sarti, A., Tubaro, S.: TDOA-Based Acoustic Source Localization in the Space-Range Reference Frame, Multidimension. Syst. Signal Process. 25, 2013, 337-359.

[3] Coll, B., Ferrando, J., Morales-Lladosa, J.: Positioning systems in Minkowski space-time: Bifurcation problem and observational data, Phys. Rev. D 86 (8), 2012, 084036.

[4] Compagnoni, M., Notari, R., Antonacci, F., Sarti, A.: A comprehensive analysis of the geometry of TDOA maps in localization problems, Inverse Prob. 30 (3), 2014, 035004.

[5] Huang, Y., Benesty, J.: Audio Signal Processing for Next Generation Multimedia Communication Systems Kluwer Academic Publishers, 2004.

\section{Xray microtomography at Elettra \\ Diego Dreossi, Elettra - Sincrotrone, Trieste}

The talk by Diego Dreossi focused on the description of the research activity developed at Elettra Sincrotrone Trieste.

It is a multidisciplinary laboratory, specialized in generating high quality synchrotron light and applying it in materials science. The facility is open to external users and every year scientists and engineers from more than 50 different countries compete by submitting proposals for the access to it.

One of the beamlines, SYRMEP, is dedicated to the imaging with hard X-rays (8-38 keV) exploiting all the features of the beam (coherence, monochromaticity, geometry). Several imaging modalities are available both for planar radiography and tomography and a complementary microfocus based CT facility was developed to extend the capabilities of the center.

The large variety of samples ranging from geology to food science, from paleontology to biology, from wood technology to material science shows the growing interest in CT from the world of scientific and applied research. The CT acquisition and reconstruction is only the first step in a long process towards a synthetic answer to a specific question, but it is of fundamental importance.

The SYRMEP experience has been presented with some illustrative examples. 


\section{References}

[1] Baker, D.R., Brun, F., O’Shaughnessy, C., Mancini, L., Fife, J.L., Rivers, M.: A four-dimensional X-ray tomographic microscopy study of bubble growth in basaltic foam, Nat. Commun. 3, 2012, 11-35.

[2] Bernasconi, A., Cosmi, F., Dreossi, D.: Local anisotropy analysis of injection moulded fibre reinforced polymer composites, Compos. Sci. Technol. 68 - 12, 2008, 2574-2581.

[3] Brun, F., Mancini, L., Kasae, P.,. Favretto, S, Dreossi, D., Tromba, G.: Pore3D: A software library for quantitative analysis of porous media,Nucl. Instrum. Methods Phys. Res., Sect. A 615 - 3, 2010, 326-332.

[4] Castelli, E., Tonutti, M., Arfelli, F., Longo, R., Quaia, E., Rigon, L., Sanabor, D., Zanconati, F., Dreossi, D., Abrami, A., Quai, E., Bregant, P., Casarin, K., Chenda, V., Menk, R.H., Rokvic, T., Vascotto, A., Tromba, G., Cova, M.A.: Mammography with Synchrotron Radiation: First Clinical Experience with Phase-Detection Technique Radiol. 259 - 3, 2011, 684-694.

[5] Sodini, N., Dreossi, D., Chen, R., Fioravanti, M., Giordano, A., Herrestal, P., Rigon, L., Zanini, F.: Noninvasive microstructural analysis of bowed stringed instruments with synchrotron radiation X-ray microtomography, Journal of Cultural Heritage 13 - 3, Supplement, 2012, S44-S49.

[6] Tavella, S., Ruggiu, A., Giuliani, A., Brun, F., Canciani, B., Manescu, A., Marozzi, K., Cilli, M., Costa, D., Liu, Y., Piccardi, F., Tasso, R., Tromba, G., Rustichelli, F., Cancedda, R.: Bone turnover in wild type and pleiotrophin-transgenic mice housed for three months in the international space station (ISS), PLoS ONE 7 3, 2012.

\section{Metal Artifact Reduction in Computed Tomography Images by Fourth-Order Variational Inpainting Methods Elena Faggiano, Politecnico di Milano}

Permanent metallic implants such as dental fillings, hip prostheses and cardiac devices generate streakslike artifacts in computed tomography images. During the past three decades, various Metal Artifact Reduction (MAR) techniques have been proposed for reducing such artifacts. Among MAR techniques, interpolation-based methods aim at identifying the corrupted parts of the sinogram and restoring them using information coming from the uncorrupted neighboring projections, for example through linear interpolation LI or inpainting techniques. In this presentation Elena Faggiano proposed two methods to reduce metal artifacts, both based on partial differential equations (PDEs): the Cahn-Hilliard equation and the TV-H-1 inpainting equation. Although already profitably employed in other branches of image processing, these two fourth-order variational methods have never been used to perform metal artifact reduction. A systematic evaluation of the performances of the two methods has been carried out. Comparisons have been provided with the results obtained through classical linear interpolation and two other PDE-based approaches using, respectively, the Fourier heat equation and a nonlinear version of the heat equation relying on total variation flow. Visual inspection of both synthetic and real computed tomography images, as well as computation of similarity indexes, suggest that the Cahn-Hilliard method behaves comparably to more classical approaches, whereas the TV- H-1 method outperforms the others as it provides best image restoration, highest similarity indexes and it is the only method able to recover hidden structures, a task of primary importance in the medical field. 


\section{References}

[1] Burger, M., He, L., Schönlieb, C.B.: Cahn-hilliard inpainting and a generalization for grayvalue images, SIAM J. Imag. Sci. 2 - 4, 2009, 1129-1167.

[2] Chen, Y., Li, Y., Guo, H., Hu, Y., Luo, L., Yin, X., Gu, J., Toumoulin, C.: Ct metal artifact reduction method based on improved image segmentation and sinogram inpainting, Mathematical Problems in Engineering, 2012 ID 786281.

[3] Duan, X. , Zhang, L., Xiao, Y., Cheng, J., Chen, Z., Xing, Y.: Metal artifact reduction in ct images by sinogram tv inpainting, In Nuclear Science Symposium Conference Record, NSS08, IEEE, 2008, 4175-4177.

[4] Meyer, E., Raupach, R., Schmidt, B., Mahnken, A.H., Kachelrie, M.: Adaptive normalized metal artifact reduction (anmar) in computed tomography, In Nuclear Science Symposium and Medical Imaging Conference (NSS/MIC), IEEE, 2011, 2560-2565.

[5] Schönlieb, C.B., Bertozzi, A.: Unconditionally stable schemes for higher order inpainting, Commun. Math. Sci., 9 - 2, 2011, 413-457.

\section{Background Information Noise Characterization for Discrete Tomography Application (Whiter than white data) Rodolfo Fiorini, Politecnico di Milano}

Discrete tomography (DT) focuses on the case where only few specimen projections are known and the images contain a small number of different colors (eg black-and-white). In this case, conventional reconstruction techniques all fail. Yet, several practical applications demand accurate reconstructions under these conditions. The reconstruction of images using more than two gray-levels, or the reconstruction of three-dimensional images are currently under consideration. On the other hand, the general discipline of computational tomography (CT) has been used over the past 40 years, particularly in medical imaging (CAT-scanners), for making noninvasive images of patients. When a large number of projections (X-rays) is available, accurate reconstructions can be made by a wide spectrum of available methods. Applications of these methods are, among others, radiology (CT-, MRI- and PET- scans), geophysics and material science. The tomographic problems can be formulated as a system of linear equations. Unfortunately, these systems are not symmetric nor positive (semi)definite, rank deficient and not square. Furthermore, with every different kind of CT, and thus also with DT, one is faced with experimental noisy data. Because of this background noise in the data, the reconstruction process is more difficult since the system of equations becomes easily inconsistent. In fact the discrete inverse problem of reconstructing finite subsets of the n-dimensional integer lattice $Z^{n}$ that are only accessible via their line sums (discrete $\mathrm{X}$-rays) in a finite set of lattice directions usually results into an even more ill-posed problem from data obtained from noisy experimental environment. Therefore, a tool able to capture and reliably classify the subtle background noise characteristics of any real experimental tomography set-up could be quite useful to get better overall result by data cleaning. In his talk Rodolfo Fiorini critically discussed a first attempt to develop such a tool by means of a combinatorial approach, comparing it to standard stochastic techniques. Two practical examples have been presented and commented. 


\section{References}

[1] Bracewell, R.N. , Riddle, A.C.: Inversion of fan-beam scans in radio astronomy, Astrophys. J. 150, 1976, 427-434.

[2] Dorairangasamy, M.A., Mredhula, L.: An Extensive Review of Significant Researches on Medical Image Denoising Techniques, Int. J. Comput. Appl. Technol. 64 - 14, 1-12.

[3] Fiorini, R.: Strumentazione Biomedica: Sistemi di Supporto Attivo, CUSL, Collana Scientifica, Milano (1994).

[4] Fiorini, R., Laguteta, G., Discrete Tomography Data Footprint Reduction by Information Conservation, Fundam. Inf. 125, 2013, 261-272.

[5] Pascal, V.: Numerical Simulation in Statistical Physics, Lecture in Master 2, Physics of complex systems" and Modeling, Statistics and Algorithms for out-of-equilibrium systems, Laboratoire de Physique Thorique de la Matire Condense Paris 2012.

[6] Shannon, C.E.: A Mathematical Theory of Communication, Bell System Technical Journal 27 - 31948 , $379-423$.

\section{On Additivity and Fixing Numbers of Matrices: Uniqueness in Discrete Tomography Barbara Langfeld, Christian-Albrechts-Universitat zu Kiel}

The talk by Barbara Langfeld surveyed results from [5] concerning substructure uniqueness and uniqueness induced by substructures.

Let us recall that for substructure uniqueness the reconstruction and uniqueness problem from discrete tomography can be translated to the language of integer linear programming:

$$
A x=b \quad \wedge \quad 0 \leq x \leq \mathbb{1}_{q} \wedge x \in \mathbb{Z}^{q},
$$

where $q$ is the number of grid points, $b=\left(\beta_{1}, \ldots, \beta_{p}\right)^{T} \in \mathbb{R}^{p}$ encodes the $\mathrm{X}$-ray measurements for all $p$ $\mathrm{X}$-ray lines, and $A=\left(\alpha_{i, j}\right) \in \mathbb{R}^{p \times q}$ lists if grid point $j$ contributes to X-ray line $i$.

The concept of $J$-additivity for a solution $x^{*}=\left(\xi_{1}, \ldots, \xi_{q}\right)^{T} \in\{0,1\}^{q}$ of (ILP), for $J \subseteq\{1, \ldots, q\}$, has been introduced and discussed, showing that it is a sort of weighted, filtered back-projection, that, for $J=\{1, \ldots, q\}$, gives the classical notion of additivity [1,3]. In fact, this seemingly 'complex' notion proves to be easily described via linear programming duality with the aid of the polytopes $P:=\{x \in$ $\left.\mathbb{R}^{q}: A x=b \wedge 0 \leq x \leq \mathbb{1}_{q}\right\}$ and $P_{J}:=P_{J}\left(x^{*}\right):=P \cap\left\{\left(\xi_{1}, \ldots, \xi_{q}\right)^{T} \in \mathbb{R}^{q}:\left(\xi_{j}^{*}\right)_{j \in J}=\left(\xi_{j}\right)_{j \in J}\right\}$.

Theorem 1. $P=P_{J}$ if and only if $x^{*}$ is $J$-additive. In particular, $P=\left\{x^{*}\right\}$ if and only if $x^{*}$ is additive.

So $J$-additivity of $x^{*}$ implies that the $J$-coordinates of $x^{*}$ are shared by any solution of (ILP).

The Theorem allows two conjectures to be disproved. Firstly, A. Kuba asked if a finite set from $\mathbb{Z}^{3}$ is uniquely determined by its X-rays along the coordinate directions if and only if it is additive. The answer is negative:

Corollary 2. There exist finite subsets of $\mathbb{Z}^{3}$ that are not additive but uniquely determined by their $X$-ray data along the three standard coordinate directions. 
Secondly, S. Brunetti and A. Daurat asked in [2] if a lattice convex set $F$ is even additive w. r. t. the set of directions $(1,0)^{T},(2,1)^{T},(0,1)^{T},(-1,2)^{T}$, which uniquely determines $F$ within the class of all finite, lattice convex subsets of $\mathbb{Z}^{2}([4])$.

Corollary 3. There exist finite sets $F, F^{\prime} \subseteq \mathbb{Z}^{2}$ such that $F$ is lattice convex, $F^{\prime}$ is not, and $F$ has the same $X$-rays along the directions $(1,0)^{T},(2,1)^{T},(0,1)^{T},(-1,2)^{T}$ as $F^{\prime}$.

Concerning uniqueness induced by substructures, revealing parts of a solution can guarantee a unique reconstruction even if the X-ray data allows for two or more solutions. However, it is hard to find a smallest possible set of such 'hints'.

Theorem 4. The following problem is $\mathbb{N P}$-complete: Given a 0-1-matrix $F$ and a number $k$, decide if there exists a set of at most $k$ of the positions of $F$ such that the disclosure of these positions and of the row and column sums of $F$ determine $F$ uniquely.

This result has interesting consequences for polytopes, as it is discussed in [5].

\section{References}

[1] Aharoni, R., Herman, G., Kuba, A.: Binary vectors partially determined by linear equation systems, Discrete Math. 171, 1997, 1-16.

[2] Brunetti, S., Daurat, A.: Stability in discrete tomography: Some positive results. Discrete Appl. Math. 147, 2005, 207-226.

[3] Fishburn, P., Lagarias, J., Reeds, J., Shepp, L.: Sets uniquely determined by projections on axes. II: Discrete case. Discrete Math. 91, 1991, 149-159.

[4] Gardner, R., Gritzmann, P.: Discrete tomography: Determination of finite sets by X-rays. Trans. Amer. Math. Soc. 349, 1997, 2271-2295.

[5] Gritzmann, P., Langfeld, B., Wiegelmann, M.: Uniqueness in discrete tomography: Three remarks and a corollary. SIAM J. Discrete Math. 25 - 4 ,2011, 1589-1599.

\section{Projection ghosts in periodic and non-periodic spaces Nicolas Normand, University of Nantes}

For several years, the research group of Nicolas Normand has explored applications of discrete tomography to information representation for transmission and distributed storage [3]. The initial investigation has been based on the non-periodic Mojette Transform (MT), a discrete version of the Radon transform, where projection angles are given by a pair of coprime integers. More recently the Finite Radon Transform (FRT) has been employed, and defined in a periodic space. The FRT and, in some cases the MT, can be summarized by a Vandermonde matrix with rows in direct and transform spaces represented by polynomials [4]. This algebraic representation leads to new algorithms or to a new interpretation of existing ones. For example, the talk included an adaptation of Björck and Pereyra's Vandermonde system solving algorithm [1] for both the FRT and the MT.

Projection ghosts, also known as switching components, are non-null images whose projections cancel along a set of directions, making them invisible. By the linearity of the projection operator it is clear 
that, for a collection of angles, uniqueness of reconstruction of a data set is equivalent to the nonexistence of ghosts. Determining ghosts presence is thus essential to unambiguous information representation. But beyond this uniqueness criterion, ghosts also play a role in reconstruction algorithms of data sets when some projections are null by suitable constraints [2].

Particular ghosts, that vanish in $n$ directions while containing only $2 n$ points ( $n$ "pluses" and $n$ "minuses"), are extremely interesting. By minimizing the count of non-zero points, they may lead to more efficient implementations. Their combinatorial structure is similar to that of Latin squares, and they were first investigated from that point of view. Lately, a more systematic construction of FRT minimal ghosts was proposed that uses a transformation matrix which is a $n$-th root of identity [5].

\section{References}

[1] Björck, A., Pereyra, V.: Solution of Vandermonde systems of equations, Mathematics of Computation, 24(112), October 1970, 893-903.

[2] Chandra, S., Svalbe, I. D., Guédon, J., Kingston, A. M., Normand, N.: Recovering Missing Slices of the Discrete Fourier Transform using Ghosts, IEEE Transactions on Image Processing, 21(10), October 2012, 4431-4441.

[3] Guédon, J., Ed.: The Mojette Transform: Theory and Applications, Wiley-ISTE, January 2009, ISBN 9781848210806.

[4] Normand, N., Svalbe, I. D., Parrein, B., Kingston, A. M.: Erasure Coding with the Finite Radon Transform, Wireless Communications \& Networking Conference, IEEE, Sydney, April 2010.

[5] Svalbe, I. D., Normand, N.: Properties of Minimal Ghosts, Discrete Geometry for Computer Imagery 2011 (I. Debled-Rennesson, É. Domenjoud, B. Kerautret, P. Even, Eds.), 6607, Springer Berlin / Heidelberg, Nancy, France, April 2011, ISBN 978-3-642-19866-3.

\section{Pattern Avoiding Polyominoes \\ Simone Rinaldi, Universitá di Siena}

One of the most investigated and fruitful notions in contemporary combinatorics is that of a pattern. Historically it was first considered for permutations, then analogous definitions were provided in the context of many other structures, such as set partitions, words, trees, and paths.

The talk by Simone Rinaldi focused on the notion of pattern in the context of polyominoes. He reported on a work which begins the systematic study of such a notion. The aim is that of translating on pattern avoiding polyominoes most of the properties of pattern avoiding permutations. Similarly to the case of permutations, the pattern-containment relation defines a poset structure on the set of all polyominoes.

For a given set $S$ of patterns, let $A V(S)$ be the set of polyominoes avoiding $S$. Two sets of patterns $S$ and $S^{\prime}$ are equivalent if $A V(S)=A V\left(S^{\prime}\right)$. As a first result it was shown that for every set $S$ of matrix patterns there is a set $P(S)$ of polyomino patterns equivalent to $S$.

Then a definition of stability has been provided for a class of pattern avoiding polyominoes. A class $U$ of permutations is stable if and only if, for every permutation $P$ in $U$, every pattern contained in $P$ is still an element of $U$. Stability is a remarkable property within permutations since we have that a class of permutation $U$ is stable if and only if there is a set $S$ of patterns such that $U=A V(S)$. Stable classes of polyominoes have been presented, together with their combinatorial and geometrical properties. 
Finally, for a given class $O$ of polyominoes, it was shown that a set $S$ of patterns exists such that $O=A V(S)$. The most of the known classes of polyominoes can be expressed as pattern avoiding polyominoes, such as parallelogram polyominoes, directed convex polyominoes, stack polyominoes, Ferrers diagrams, directed polyominoes, column-convex polyominoes, and L-convex polyominoes. Moreover, the class of polyominoes which are uniquely determined by their horizontal and vertical projections can be expressed as a class of pattern avoiding polyominoes.

On the other side, various types of simple patterns have been considered, and for each pattern $S$, the class $A V(S)$ has been characterized and studied, providing its combinatorial properties in relation to other areas of discrete mathematics, discrete tomography and computational geometry.

\section{References}

[1] Bona, M.: Combinatorics of permutations Chapman-Hall and CRC Press (2004).

[2] Brändén, P., Claesson, A.: Mesh patterns and the expansion of permutation statistics as sums of permutation patterns, Electron. J. Comb., 18 - 2, $\sharp$ P5, 2011-2 (The Zeilberger Festschrift volume)

[3] Castiglione, G., Restivo, A.: Ordering and Convex Polyominoes, in: M. Margenstern (Ed.), Machines, Computations, and Universality 2004, Saint Petersburg, Russia, Lecture Notes in Computer Science 3354, Springer 2005.

\section{Differential properties of u-maps}

Grzegorz Sójka, Warsaw University of Technology

It is known that all convex bodies are uniquely determined by certain sets of four X-rays (see 1.2.12 in [1]). Thus, it is natural to ask how to find a convex body knowing only the values of its X-ray functions. R. J. Gardner in [1] suggested an idea of exact reconstruction method (see 1.2.24-1.2.28). The open problem is whether his method always gives us full information about the original body. This actually leads to considering 1-dimensional dynamical system generated by non-abelian group. In his talk Grzegorz Sójka presented some partial results concerning this problem.

\section{References}

[1] Gardner, R.J., Geometric Tomography, Cambridge University Press 1995, (second edition 2006).

\section{Polarimetric SAR Tomography for Three-dimensional Imaging of Natural Media Stefano Tebaldini. Politecnico di Milano}

Synthetic Aperture Radar (SAR) is a remote sensing technology that allows to obtain images of the Earths surface by transmitting and receiving microwave pulses. A massive amount of data has nowadays been produced by a number of dedicated spaceborne missions, as well as ground based and airborne surveys carried out every year, allowing accurate monitoring of the Earths surface and retrieval of different geophysical parameters. The employment of microwaves makes SAR images quite different from optical ones for a number of reasons. For the aim of this paper the most relevant difference with respect to optical frequencies is that microwaves have the ability to penetrate the illuminated media, hence providing 
sensitivity about the inner layers. This is the case of forests, snowpack, ice sheets, and arid terrains. In recent years much effort has been paid to fully exploit this potential by developing system configurations as well as processing methods for the three dimensional reconstruction of the electromagnetic field scattered by the illuminated objects, giving rise to a nowadays acknowledged research field commonly referred to as SAR Tomography. Moreover, SAR remote sensing also provides the possibility to infer information about the illuminated scene by varying wave polarization at transmission and reception, which results in signal variations that are linked to the physical properties of the targets. The joint exploitation of polarimetric and tomographic SAR (Polarimetric SAR Tomography) data provides in depth analysis of the 3D structure of the investigated scenes, revealing precise information of the different scattering mechanisms ruling the interaction of Radar waves with the illuminated objects. The talk provided an overview of the basic principles and processing methods peculiar to SAR Tomography and Polarimetric SAR Tomography. It focused on joint work of Stefano Tebaldini with Fabio Rocca, Ho Tong Minh Dinh, Mauro Mariotti dAlessandro, Francesco Banda. A few case studies have been presented relative to tomographic and polarimetric/tomographic analysis of boreal forests, tropical forests, ice, and snowpack.

\section{References}

[1] D’Alessandro, M.M., Tebaldini, S., Rocca, F.: Phenomenology of Ground Scattering in a Tropical Forest Through Polarimetric Synthetic Aperture Radar Tomography, IEEE Trans. Geosci. Remote Sens. 51, 8, 4430,4437, Aug. 2013

[2] Dinh, H.T.M., Tebaldini, S., Rocca, F., Koleck, T., Borderies, P., Albinet, C., Villard, L., Hamadi, A., Toan, T.L.: Ground-Based Array for Tomographic Imaging of the Tropical Forest in P-Band, IEEE Trans. Geosci. Remote Sens. 51, 8, 4460,4472, Aug. 2013,

[3] Tebaldini, S.: Algebraic Synthesis of Forest Scenarios From Multibaseline PolInSAR Data, IEEE Trans. Geosci. Remote Sens. 47, 12, 4132-4142, Dec. 2009

[4] Tebaldini, S., Rocca, F.: Multibaseline Polarimetric SAR Tomography of a Boreal Forest at P- and L-Bands, IEEE Trans. Geosci. Remote Sens. 50, 1, 232-246, Jan. 2012,

\section{Estimates for solutions of binary tomography problems Robert Tijdeman, Leiden University}

Let a bounded domain $D$ in the plane $\mathbb{R}^{2}$ be given. Suppose that the area of $D$ in parallel beams of width $w$ is measured in $k$ directions. The task is to find a function $f$ on the integer lattice $\mathbb{Z}^{2}$ such that the corresponding measurements are close to those of $D$. In his talk Robert Tijdeman presented an estimate of how well the task can be performed. Particular attention has been given to estimates which are independent of the size of $D$. Several links with the literature $[1,2,3,4,5]$ have been provided.

\section{References}

[1] Batenburg, K.J., Fortes, W., Hajdu, L., Tijdeman, R.: Bounds on the difference between reconstructions in binary tomography, Discrete Geometry and Computer Imaginary LNCS 6607, 2011, 369-380.

[2] Batenburg, K.J., Fortes, W., Tijdeman, R.: Approximate discrete reconstruction algorithms, Fundam. Inform. $125,2013,239-259$. 
[3] van Dalen, B., Hajdu, L., Tijdeman, R.: Bounds for discrete tomography solutions, Indag. Math., 24, 2013, 391-402.

[4] Hajdu, L., Tijdeman, R.: Algebraic aspects of discrete tomography, J. Reine Angew. Math., 534, 2001, 119128.

[5] Hajdu, L., Tijdeman, R.: Bounds for approximate tomography solutions, SIAM J. Discr. Math. 27, 2013, 1055-1066.

Acknowledgements. We kindly thank all the authors of the papers published in this special issue as well as all the referees who devoted their precious time to produce thorough reviews providing valuable comments and suggestions for improving the submitted manuscripts. Many thanks also to the speakers of the 7th Meeting on Tomography and Applications for their interesting lectures. We wish to thank the agencies supporting the Meeting: Department of Mathematics- Politecnico di Milano, Università Cattolica di Piacenza, and MIUR- PRIN 2010-2011 Automi e Linguaggi Formali: Aspetti Matematici e Applicativi. A special gratitude goes to Professor Damian Niwiński, who gave us the opportunity of publishing this special issue in Fundamenta Informaticae.

\section{Paolo Dulio}

Dipartimento di Matematica "F. Brioschi"

Politecnico di Milano, Italy

paolo.dulio@polimi.it

\section{Andrea Frosini}

Dipartimento di Sistemi e Informatica

Universitâ di Firenze, Italy

andrea.frosini@unifi.it

\section{Grzegorz Rozenberg}

LIACS, Leiden University, The Netherlands and

Department of Computer Science

University of Colorado at Boulder, USA

g.rozenberg@liacs.leidenuniv.nl

(Editors of the issue) 